\title{
Invasive breast cancer induces laminin-332 upregulation and integrin $\beta 4$ neoexpression in myofibroblasts to confer an anoikis-resistant phenotype during tissue remodeling
}

Baek Gil Kim', Ming-Qing Gao², Yoon Pyo Choi', Suki Kang ${ }^{3,4}$, Haeng Ran Park', Kyu Sub Kang ${ }^{1}$ and Nam Hoon Cho ${ }^{1,2,3,4^{*}}$

\begin{abstract}
Introduction: Although development of anoikis-resistant myofibroblasts during tissue remodeling is known to be associated with tumor invasion, the mechanism by which myofibroblasts become resistant to anoikis is unknown. We previously demonstrated laminin-332 upregulation in the fibrosis around invasive ductal carcinoma (IDC). Because laminin-332 promotes cell survival through binding to integrins, we hypothesized that invasive breast cancer cells confer an anoikis-resistant phenotype on myofibroblasts by upregulating laminin-332 expression during tissue remodeling. Here, we demonstrate that invasive breast cancer cells induce laminin-332 upregulation and integrin $\beta 4$ neoexpression in myofibroblasts to confer an anoikis-resistant phenotype.

Methods: Three types of fibroblasts were isolated from the tumor burden, the fibrosis, and normal tissue of patients with early stage IDC (less than $10 \mathrm{~mm}$ diameter), designated cancer-associated fibroblasts (CAFs), interface fibroblasts (InFs), and normal breast fibroblasts (NBFs), respectively. To investigate direct and indirect crosstalk with tumor cells, fibroblasts were co-cultured with invasive MDA-MB-231 or noninvasive MCF7 cells or in conditioned medium. Anoikis resistance of fibroblasts was measured by cell viability and caspase-3 activity after incubation on poly-HEMA coated plates for 72 hours. Involvement of laminin-332/integrin $\alpha 3 \beta 1$ or $\alpha 6 \beta 4$ signaling in anoikis resistance was confirmed by treatment with purified laminin-332 or blocking antibodies against laminin-332, integrin $\beta 1$, or integrin $\beta 4$.
\end{abstract}

Results: MDA-MB-231 cells induced laminin-332 upregulation and integrin $\beta 4$ neoexpression in fibroblasts, leading to anoikis resistance. InFs showed a higher endogenous level of laminin-332 than did CAFs and NBFs. After stimulation with MDA-MB-231-conditioned medium, laminin-332 expression of InFs was dramatically increased and maintained under anoikis conditions. Laminin-332 upregulation was also observed in CAFs and NBFs, but at a lower level than in InFs. Laminin-332 induced Akt (Ser473) phosphorylation by binding to integrin $\alpha 3 \beta 1$. Integrin $\beta 4$ neoexpression induced laminin-332-independent Rac1 activation and promoted anoikis resistance in fibroblasts approximately twofold more effectively than did laminin-332, regardless of the type of fibroblast. In addition, integrin $\beta 4$ expression suppressed fibroblast aggregation in conditions of anoikis.

Conclusion: Invasive breast cancer cells confer an anoikis-resistant phenotype on myofibroblasts during tissue remodeling by inducing laminin-332 upregulation and integrin $\beta 4$ neoexpression. Interface fibroblasts appear to be the primary myofibroblasts that interact with invasive tumor cells during tissue remodeling.

\footnotetext{
* Correspondence: cho1988@yuhs.ac

'Brain Korea 21 Project for Medical Science, Yonsei University College of

Medicine, 134 Sinchon-dong, Seodaemun-gu, Seoul, 120-752, South Korea

Full list of author information is available at the end of the article
}

(c) 2012 Cho et al.; licensee BioMed Central Ltd. This is an open access article distributed under the terms of the Creative Commons 


\section{Introduction}

A fundamental component of tumor invasion is stromal tissue remodeling, which involves proteolytic degradation of the extracellular matrix (ECM) and results in anoikis (a form of caspase-dependent apoptosis that is caused by loss of integrin binding of stromal cells) [1] As a component of stroma, myofibroblasts are also exposed to anoikis during tissue remodeling. However, many studies have reported prolonged survival of myofibroblasts during tissue remodeling in patients with fibrotic diseases [2-5]. Fibrosis is considered an indicator of tissue remodeling [6] and is commonly formed around invasive types of tumors [7-9]. Considering that myofibroblasts are key regulators of tissue remodeling [10] and a major source of ECM production [11], which drives tumor progression, the development of anoikisresistant myofibroblasts may be an essential event during stromal tissue remodeling before tumor invasion.

Abnormal and excessive ECM deposition not only is a phenotype of fibrosis but also is associated with cell-survival signaling mediated by integrin receptors during tumor invasion and tissue remodeling [12]. Therefore, altered molecular expression in fibrosis may provide a clue to how myofibroblasts acquire an anoikis-resistant phenotype during tissue remodeling. Previously, we observed aberrant laminin-332 upregulation in the fibrosis of invasive ductal carcinoma (IDC) compared with autologous tumor burden and distal normal tissue, whereas such laminin-332 upregulation was not found in the noninvasive counterpart, ductal carcinoma in situ (DCIS) [13]. Contrary to our finding, laminin-332 expression was previously reported to be downregulated in breast cancer [14]. Laminin-332, a large multidomain molecule involved in cell adhesion and matrix assembly, plays an important role in cell migration and survival by activating many signal mediators through binding to integrin $\alpha 3 \beta 1$ or $\alpha 6 \beta 4$ [15-18]. The role of laminin-332 in cell survival or anoikis resistance has been studied mostly in epithelial tumor cells, although some data exist on laminin-332-dependent survival of keratinocytes during wound healing $[19,20]$. One group demonstrated that the matricellular protein thrombospondin1 (TSP1) induces anoikis resistance in mouse embryonic fibroblasts in vitro [21]. This work provided convincing evidence that an ECM protein can induce anoikis resistance in fibroblasts and suggested that laminin-332 may fulfill a similar function. However, because the fibroblasts used in previous studies were not derived from tumor stroma, the role of anoikis resistance of myofibroblasts in the context of tumor invasion remains undetermined.

In this study, we hypothesized that invasive breast cancer cells induce laminin-332 upregulation in myofibroblasts undergoing tissue remodeling, which leads to anoikis resistance through laminin-332 autocrine signaling. To test our hypothesis, we used three types of fibroblasts isolated from IDC tissue and co-cultured them with breast cancer cells to investigate tumor-stroma crosstalk. Our results provide evidence that anoikis-resistant myofibroblasts develop during tissue remodeling of invasive breast cancer as a result of abnormal upregulation of ECM proteins and/or their receptors.

\section{Materials and methods \\ Isolation of primary fibroblasts}

Human breast tumors and autologous normal breast tissues were obtained from three IDC patients undergoing surgery at Severance Hospital of the Yonsei University Health System, Korea. The protocol for the study was approved by Severance Hospital Ethics Committee (42008-0380). All participants signed written informed consent forms detailing tissue use for comprehensive experiments on breast cancer. Based on the zonal concept proposed in our previous study, primary fibroblasts were isolated from tissue in the tumor zone (TZ, tumor burden), interface zone (IF, fibrosis), and normal zone (distal normal tissue), as described previously [22].

\section{Direct and indirect co-culture}

For direct co-culture, primary fibroblasts were cultured with MCF7 or MDA-MB-231 breast cancer cells (Korean Cell Line Bank, Seoul, Korea), as described previously [23]. MDA-MB-231 and MCF7 cells were selected as representative invasive and noninvasive types of breast cancer, respectively. Adherent fibroblasts were stained with $5 \mu M$ Cell Tracker Green CMFDA (5chloromethylfluorescein diacetate; Invitrogen, Eugene, OR, USA) by incubation at $37^{\circ} \mathrm{C}$ for 45 minutes. The dye solution was then replaced with fresh prewarmed medium, and the cells were incubated at $37^{\circ} \mathrm{C}$ for another 2 hours. After washing them twice with PBS, unstained cancer cells were plated onto a layer of CMFDA-stained fibroblasts. The co-cultures were incubated in reduced serum media (RSM) composed of DMEM/F12, 1\% FBS, $100 \mathrm{IU} / \mathrm{ml}$ penicillin, and $100 \mu \mathrm{g} /$ $\mathrm{ml}$ streptomycin for 1 week. CMFDA-stained fibroblasts and unstained breast cancer cells were sorted with flow cytometry and used for subsequent experiments. For indirect co-culture, conditioned medium $(\mathrm{CM})$ was prepared by culturing MCF7 or MDA-MB-231 breast cancer cells at $80 \%$ confluency in RSM for 1 day. The CM was filtered through a $0.22-\mu \mathrm{m}$ filter (Millipore, Billerica, MA, USA) and diluted 1:1 with fresh RSM before addition to fibroblast cultures, which were incubated for a further 3 days. 
Transfection of fibroblasts with integrin $\beta 4$

For transient transfection with integrin $\beta 4$, fibroblasts (5 $\times 10^{5}$ cells/well) were plated on a six-well plate and transfected with $3 \mu \mathrm{g}$ pRK6 b4 plasmid DNA (Addgene plasmid 16037; Addgene, Cambridge, MA, USA; kindly donated by Dr. FG Giancotti, Cellular Biochemistry and Biophysics Program, Memorial Sloan-Kettering Cancer Center, New York, NY, USA) [24], and $3 \mu$ l MATRA (Promokine, Heidelberg, Germany) in a total volume of $200 \mu \mathrm{l}$ Opti-MEM on a Universal Magnet Plate (Promokine) for 15 minutes. Controls were transfected with 3 $\mu \mathrm{g}$ pRK5.

\section{Anoikis assays}

Fibroblasts $\left(5 \times 10^{5}\right.$ cells/well in $\left.1 \mathrm{ml}\right)$ were added to either poly-HEMA- or serum-coated wells and incubated at $37^{\circ} \mathrm{C}$ in $5 \% \mathrm{CO}_{2}$ with RSM, MCF-7 CM, or MDA-MB-231 CM for 6, 24, 48, or 72 hour. Maintenance of suspended fibroblasts in poly-HEMA-coated wells (anoikis conditions) was monitored by phase microscopy. Cell viability was measured by addition of $100 \mu$ CCK-8 (Dojindo Molecular Technologies, Inc., Rockville, MD, USA) to each well. After incubation at $37^{\circ} \mathrm{C}$ for 2 hours, fluorescence was measured with a plate reader (excitation, $530 \mathrm{~nm} /$ emission, $580 \mathrm{~nm}$ ). Fibroblasts from three IDC patients were assayed in triplicate in at least three separate experiments. For function-blocking assays, antibodies against integrin $\beta 1, \beta 4$, or laminin-332 (Millipore) were added to InF cells (which have high expression of laminin-332) with MDA-MB-231 CM and incubated at $37^{\circ} \mathrm{C}$ in a humidified $5 \% \mathrm{CO}_{2}$ incubator for 24 hours. CAF cells (which express low levels of laminin-332) were treated with purified laminin-332 (Abcam, Cambridge, England) in RSM for 24 hours.

\section{Caspase- 3 activity assay}

Caspase- 3 activity in fibroblast lysates was measured by using a caspase-3 activity kit (Becton-Dickinson-Pharmingen, San Jose, CA, USA). Fibroblasts $\left(5 \times 10^{5}\right.$ cells $)$ were plated in poly-HEMA-coated-wells with RSM, MCF7 CM, or MDA-MB-231 CM and treated with blocking antibodies or purified laminin-332 for 24 hours. Fibroblast lysates were incubated in a 96-well plate in HEPES buffer with $5 \mu \mathrm{l}$ caspase-3 fluorogenic substrate (Ac-DEVD-AMC) for 1 hour at $37^{\circ} \mathrm{C}$ in the dark. Fluorescence was measured with a plate reader (excitation, $360 \mathrm{~nm} / \mathrm{emission}, 460 \mathrm{~nm}$ ).

\section{Rac1 pull-down assay}

Suspended fibroblasts were harvested by centrifugation and lysed in $1 \times$ lysis buffer. Rac/cdc42 assay reagent (10 $\mu \mathrm{l} ; 10 \mu \mathrm{g}$ ) was added to $0.5 \mathrm{ml}$ cell lysate, and the reaction mixture was incubated by rocking gently at $4{ }^{\circ} \mathrm{C}$ for
1 hour. Agarose beads were collected by pulsing for 5 seconds in a microcentrifuge at $14,000 \mathrm{~g}$, and the supernatant was discarded. The pelleted beads were washed 3 times with $0.5 \mathrm{ml} \mathrm{Mg}^{2+}$ Lysis/Wash Buffer (MLB), resuspended in $40 \mu \mathrm{l} 5 \times$ Laemmli reducing sample buffer, boiled for 5 minutes, separated by polyacrylamide gel electrophoresis (PAGE), and then detected with immunoblot.

\section{Immunoblotting}

Suspended cells were collected by centrifugation, lysed in $100 \mu$ l Protein Extraction Solution (Intron Biotech, Seongnan-si, Korea), homogenized with a 30 -gauge needle, incubated for 30 minutes at $4^{\circ} \mathrm{C}$, and then purified by centrifugation at $13,000 \mathrm{rpm}$. After quantifying proteins in the extracts by using the Bradford method, 20 $\mu \mathrm{g}$ protein was electrophoresed on $10 \%$ Tris/glycine gels (Invitrogen, Carlsbad, CA, USA), transferred to polyvinylidine fluoride (PVDF) membranes (Millipore), and probed with primary antibodies against laminin $\gamma 2$, integrin $\alpha 3$, integrin $\alpha 6$, integrin $\beta 1$, integrin $\beta 4$, Akt, phospho(Ser473)-Akt, E-cadherin, $\beta$-catenin, GAPDH (Santa Cruz Biotechnology, Santa Cruz, CA, USA), or Rac1 (Millipore). Primary antibodies were detected with horseradish peroxidase (HRP)-conjugated secondary antibodies (Invitrogen), and visualized by using enhanced chemiluminescence reagents (Santa Cruz Biotechnology).

\section{Statistics}

Statistical significance (indicated by asterisks) was determined by using the $t$ test and ANOVA. Results were considered to be significant at $P<0.05$. All statistical analyses were performed by using SPSS version 11.5 for Windows statistical software (SPSS Inc.).

\section{Results}

Invasive breast cancer cells induce upregulation of laminin-332 expression in stromal fibroblasts

Based on the zonal concept that we previously introduced, stromal fibroblasts were isolated from each zone of IDC tissue after confirming expression of laminin- $\gamma 2$, a subunit used to identify laminin-332. Primary fibroblasts from the tumor zone (TZ), interface zone (IZ), and normal zone were designated cancer-associated fibroblasts (CAFs), interface fibroblasts (InFs), and normal breast fibroblasts (NBFs), respectively. In morphology, InFs were slightly longer than CAFs and NBFs (Figure 1A). After culture, InFs expressed a higher level of laminin- $\gamma 2$ than did CAFs and NBFs, as they do in situ, and InFs and CAFs expressed a higher level of $\alpha$-SMA did than NBFs (Figure 1B). To test whether factors secreted by invasive breast cancer cells can further increase fibroblast laminin- $\gamma 2$ expression, we treated cultured fibroblasts with 


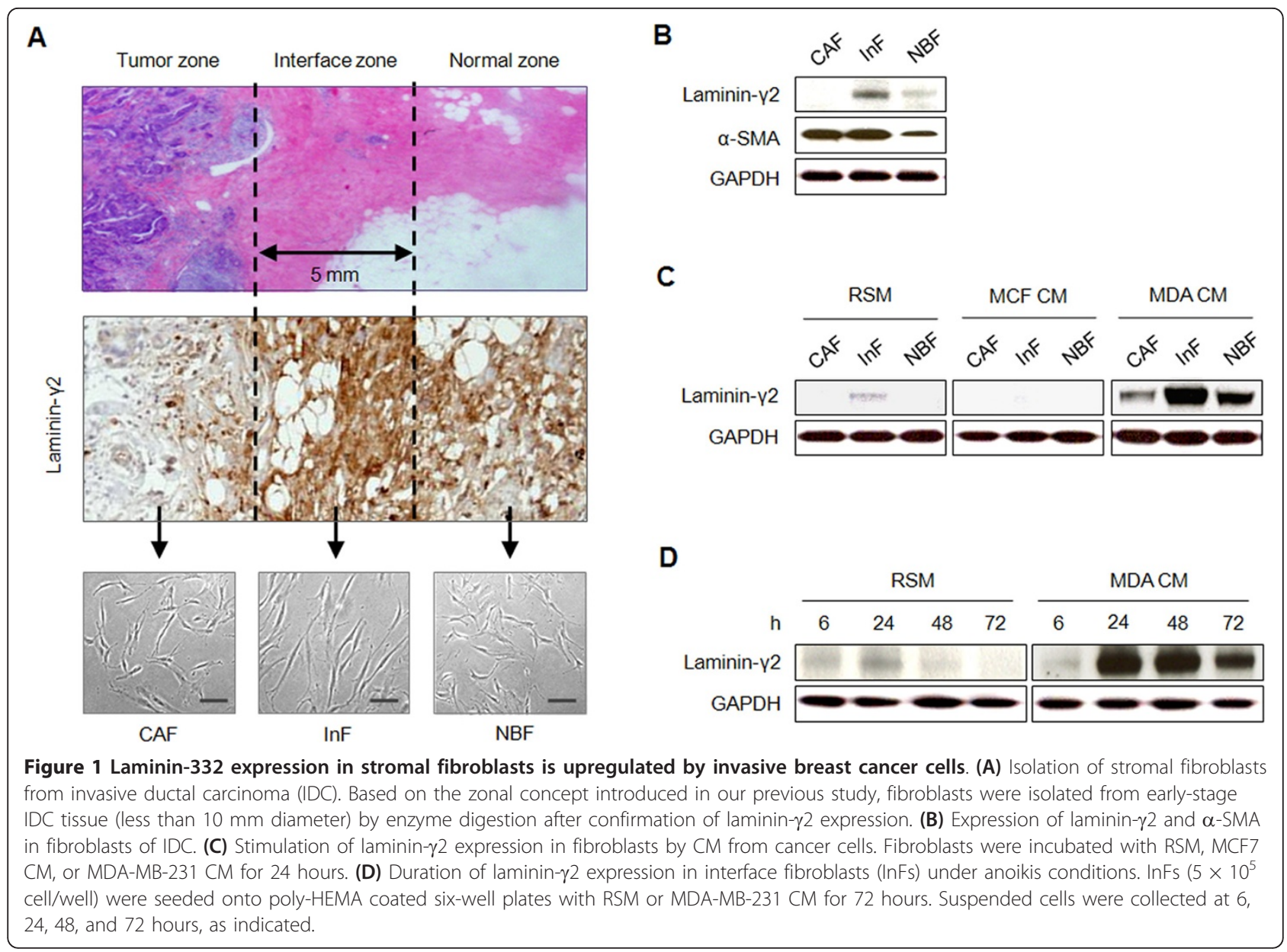

MDA-MB-231 CM. MDA-MB-231 CM dramatically increased laminin $\gamma 2$ expression in InFs compared with CAFs and NBFs. Thus, although MDA-MB-231 CM enhanced laminin $-\gamma 2$ expression in all fibroblasts, the degree of increase depended on the intrinsic capacity of the cells to express laminin- $\gamma 2$. In contrast, MCF7 CM suppressed laminin- $\gamma 2$ expression (Figure 1C). Because the IZ is densely fibrotic, a hallmark of the tissue remodeling that leads to anoikis of stromal cells, the capacity of InFs to express laminin-332 under anoikis conditions was evaluated in the absence or presence of MDA-MB231 CM stimulation. In InFs stimulated with MDA-MB$231 \mathrm{CM}$, overexpression of laminin- $\gamma 2$ was evident at 24 hours and was maintained for 72 hours (Figure 1D). Because transforming growth factor (TGF) $\beta$ is a major component of MDA-MB-231 CM, we speculated that it might be responsible for inducing laminin-332 overexpression. To test this, we treated InFs with $100 \mathrm{n} M$ LY2157299, a TGF- $\beta$ inhibitor, and MDA-MB-231 CM for 24 hours and found that laminin- $\gamma 2$ expression was suppressed in the LY2157299-treated InFs (see Additional file 1, Figure S1).
Integrin $\beta 4$ neoexpression in fibroblasts is induced through direct contact with invasive breast cancer cells Two integrin receptors for laminin-332, integrins $\alpha 3 \beta 1$ and $\alpha 6 \beta 4$, are associated with signaling pathways for cell survival. A high level of integrins $\alpha 3$ and $\alpha 6$ expression was found in the fibroblasts derived from IDC (see Additional file 1 Figure S2). Integrin $\beta 1$ was also highly expressed in the fibroblasts, whereas integrin $\beta 4$ was not expressed (Figure 2A). It is widely known that the repertoire or pattern of integrin expression in stromal cells can be changed through communication with tumor cells. Because the IZ is adjacent to the TZ within tissue, CAFs and InFs can directly or indirectly communicate with tumor cells, and, as a result, the pattern of integrin expression in myofibroblasts may be altered. To test the effect of indirect communication with tumor cells, the fibroblasts were stimulated with MDA-MB-231 CM or MCF7 CM. Integrin $\beta 1$ expression was not significantly affected, but remained high, independent of CM stimulation (see Additional file 1, Figure S3A). Integrin $\beta 4$ expression was also unchanged and remained undetectable (see Additional file 1, Figure S3B). For direct 
B

A
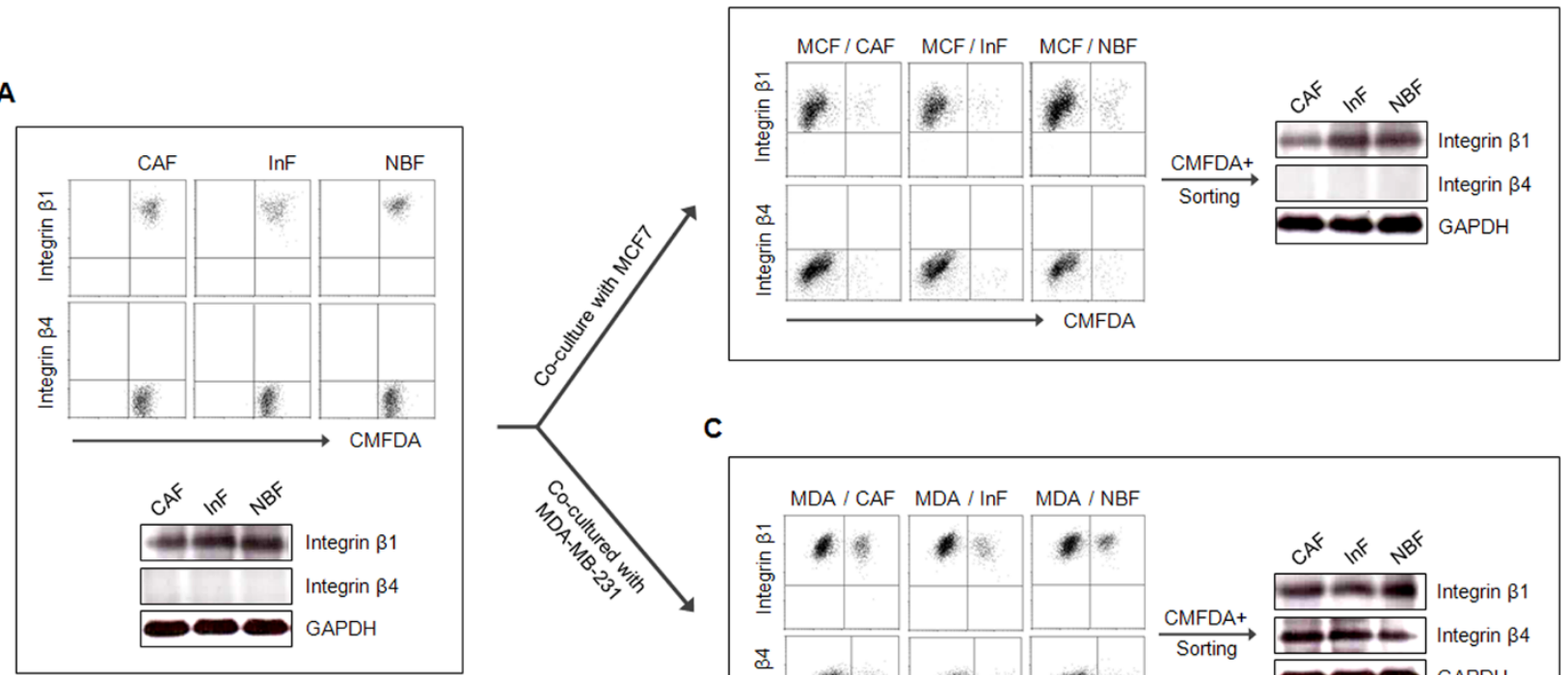

C

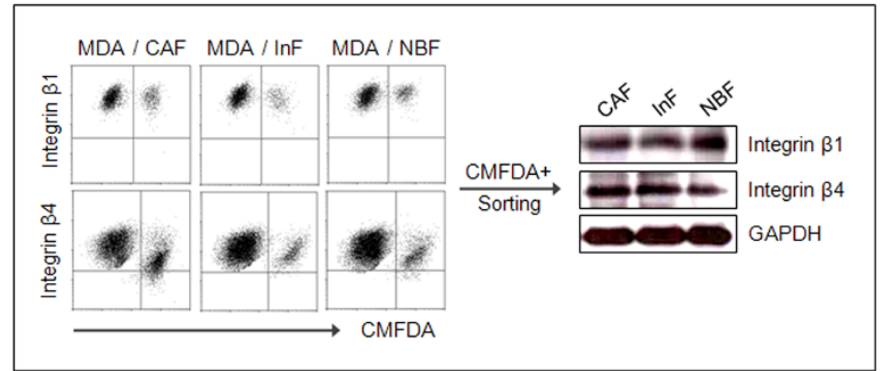

Figure 2 Integrin $\beta 4$ neoexpression is induced in fibroblasts through direct contact with invasive breast cancer cells. (A) Endogenous expression of integrin $\beta 1$ and $\beta 4$ in fibroblasts of IDC. Fibroblasts were prestained with CMFDA and cultured in RSM, MCF7 CM, or MDA-MB-231 CM for 72 hours before staining with PE-conjugated anti-integrin $\beta 1$ or $\beta 4$ antibodies. (B) Integrin $\beta 1$ expression and (C) Integrin $\beta 4$ expression in fibroblasts cocultured with MCF7 or MDA-MB-231 cells. Confluent fibroblasts on 100-mm dishes were stained with $5 \mu M$ CMFDA. MCF7 or MDA-MB-231 cells $\left(5 \times 10^{5}\right)$ were added to the stained fibroblasts and cocultured for 1 week. Mixed cells were collected and stained with PEconjugated anti-integrin $\beta 1$ or $\beta 4$ antibodies. Integrin $\beta 1$ or $\beta 4$ expression in cocultured fibroblasts was confirmed by immunoblot analysis after sorting.

communication with tumor cells, fibroblasts were cocultured with MDA-MB-231 or MCF7 cells. Integrin $\beta 1$ expression was not affected by direct contact with cancer cells, but was highly sustained (Figure 2B). In contrast, de novo expression of integrin $\beta 4$ was induced by direct contact with MDA-MB-231, but not MCF7, cells (Figure $2 \mathrm{C}$ ). Neoexpression of integrin $\beta 4$ protein was also detected in fibroblasts after sorting from the coculture.

\section{Anoikis resistance of fibroblasts is conferred by binding of laminin-332 to integrin $\alpha 3 \beta 1$ and by neoexpression of integrin $\beta 4$}

We found that MDA-MB-231 cells induced laminin-332 upregulation and integrin $\beta 4$ neoexpression in fibroblasts, and that the fibroblasts expressed a high level of integrin $\alpha 3, \alpha 6$, and $\beta 1$ independent of their interaction with cancer cells (Figure 2, and see Additional file 1, Figure S2). Therefore, we hypothesized that the fibroblasts in IDC overcome anoikis through laminin-332dependent survival pathways that are mediated by integrin $\alpha 3 \beta 1$ and/or $\alpha 6 \beta 4$. For the anoikis assay, laminin332 upregulation in fibroblasts was induced by treatment with MDA-MB-231 CM, and integrin $\beta 4$ neoexpression was induced by transfection with an integrin $\beta 4$ plasmid. Integrin $\beta 4$ overexpression was confirmed in all transfected fibroblasts (see Additional file 1, Figure S4). Anoikis resistance of fibroblasts was examined by measuring their viability under anoikis conditions. In the absence of integrin $\beta 4$ expression, the viability of InFs (which express high levels of laminin332) was increased by MDA-MB-231 CM but decreased by MCF7 CM, compared with the control incubated with reduced serum media (RSM) only. In the presence of integrin $\beta 4$ expression, the viability of InFs was dramatically increased at 6 hours and maintained at a level approximately twofold higher than that without integrin $\beta 4$ through 72 hours. In addition, the viability of these cells was further enhanced by treatment with MDAMB-231 CM (Figure 3A). Similar to that of InFs, the viability of CAFs (which express low levels of laminin332) was increased by MDA-MB-231 CM and integrin $\beta 4$ induction, although the viability of CAFs was lower than that of InFs (Figure 3B). The viability of NBFs (expressing medium levels of laminin-322) showed a pattern similar to those of InFs and CAFs (Figure 3C). The viability of the three types of fibroblasts at 72 hours is directly compared in Figure 3D. Induction of integrin 


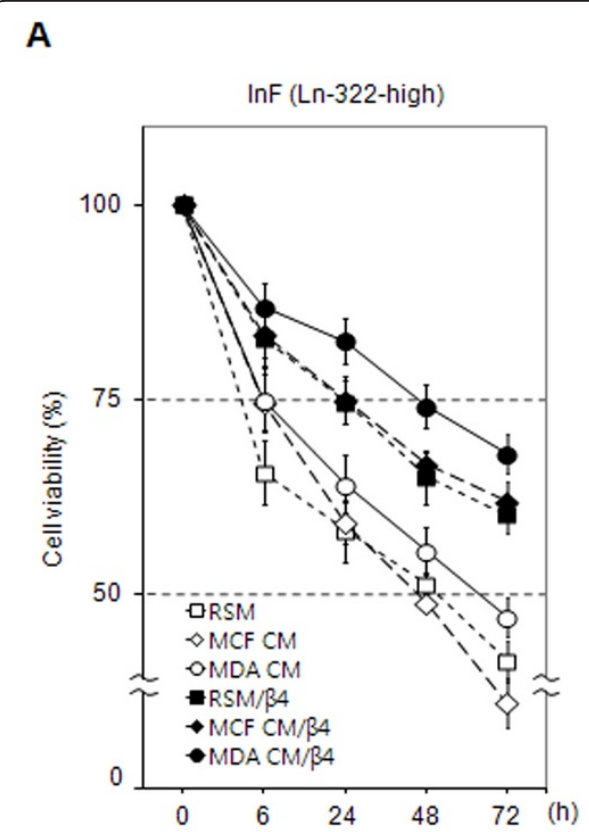

B

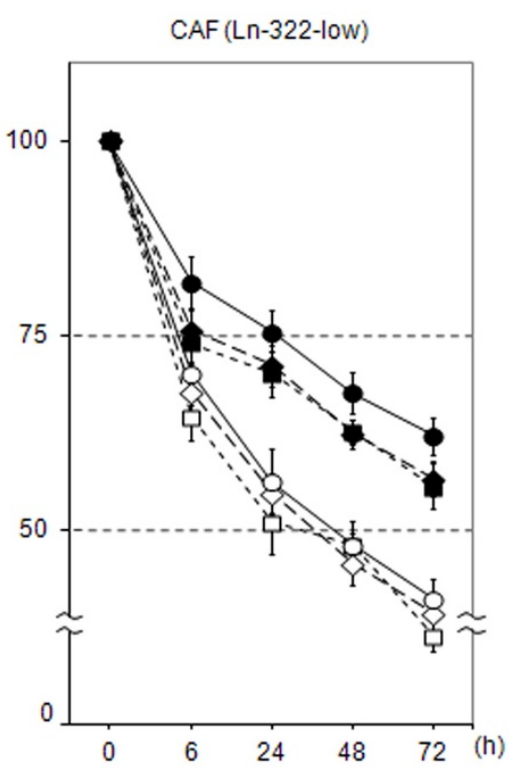

C

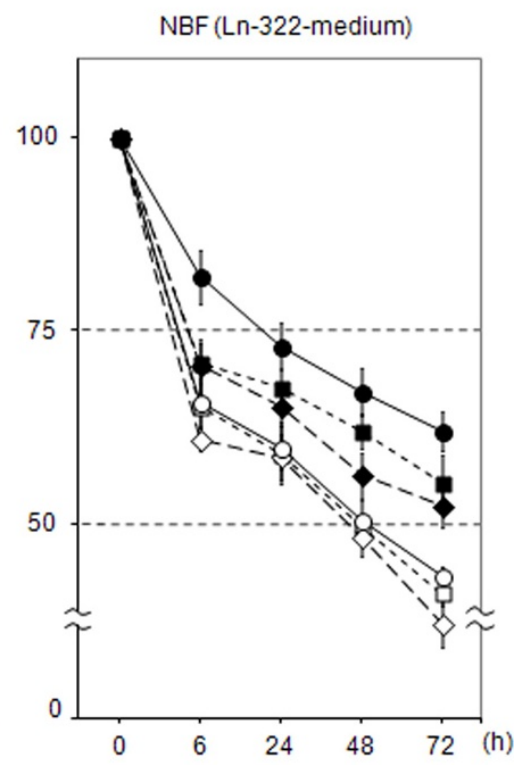

D

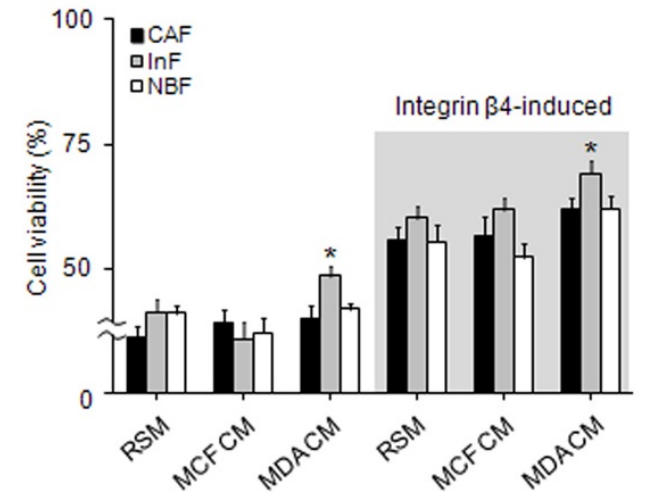

E

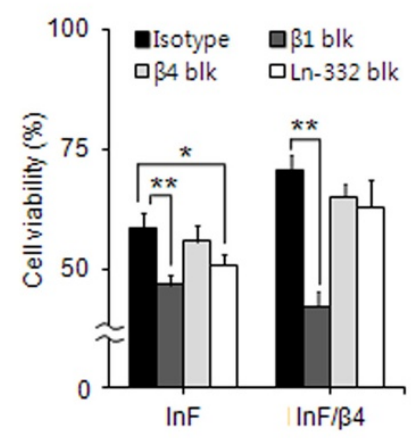

$\mathbf{F}$

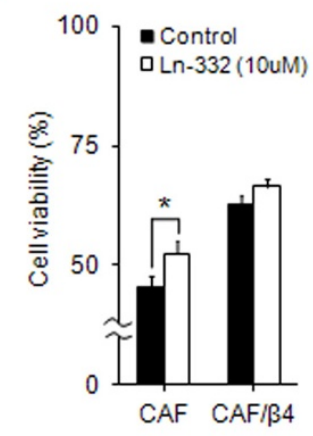

Figure 3 The anoikis resistance of fibroblasts is mediated by binding of laminin-332 to integrin $\alpha 3 \beta 1$ and/or integrin $\beta 4$ neoexpression. Viability of wild-type and integrin $\beta 4$-expressing (A) InFs, (B) CAFs, and (C) NBFs in the absence or presence of stimulation from cancer cells under anoikis conditions. The figures show fibroblast viability in RSM (open square), MCF7 CM (open diamond), and MDA-MB-231 CM (open circle) in the absence of integrin $\beta 4$ expression, and viability in RSM (black square), MCF7 CM (black diamond), and MDA-MB-231 CM (black circle) in the presence of integrin $\beta 4$ expression. (D) Direct comparison of fibroblast viabilities. The viability of wild-type and integrin $\beta 4-$ expressing fibroblasts was compared at 72 hours. Black bar, CAFs; gray bar, InFs; white bar, NBFs; gray box, integrin $\beta$ 4-expressing fibroblasts. (E) Inhibition of the interaction between laminin-332 and integrin. InF and InF/ $\beta 4$ cells were treated with isotype (black bar) and blocking antibodies against integrin $\beta 1$ (dark gray bar), $\beta 4$ (light gray bar), and laminin-332 (white bar) for 24 hours under anoikis conditions. (F) Increased viability of CAFs in response to treatment with laminin-332. CAF and CAF/ $\beta 4$ cells were treated with purified laminin-332 (diluted to $10 \mu M$ with RSM) in poly-HEMA-treated 96-well plates for 24 hours. Black bar, untreated CAFs; white bar, laminin-322-treated CAFs. Results are expressed as mean \pm SD. ${ }^{*} P<0.05 ;{ }^{* *} P<0.03$ versus RSM in poly-HEMA-coated wells. Results are averages of three separate experiments.

$\beta 4$ enhanced the viability of all fibroblast types approximately twofold compared with fibroblasts lacking integrin $\beta 4$. In contrast, MDA-MB-231 CM only increased the viability of InFs, and to a lesser extent than did integrin $\beta 4$ induction.

To confirm that fibroblast viability was associated with binding of laminin-332 to integrins $\alpha 3 \beta 1$ and/or $\alpha 6 \beta 4$, InFs and InFs/ $\beta 4$ (expressing integrin $\beta 4$ ) were first stimulated with MDA-MB-231 CM to induce laminin332 upregulation and then treated with blocking antibodies against laminin-332, integrin $\beta 1$, or integrin $\beta 4$. The viability of both $\mathrm{InF}$ and $\mathrm{InFs} / \beta 4$ was inhibited by blocking integrin $\beta 1$ and laminin-332, whereas blocking integrin $\beta 4$ had little effect (Figure 3E).

To show that the viability of fibroblasts depends on laminin-332 expression, CAFs (Ln-332-low) were treated 
with $10 \mu M$ purified laminin-332, resulting in increased viability. The viability of CAFs $/ \beta 4$ was strongly maintained, independent of laminin-332 treatment (Figure 3F).

\section{Laminin-332 upregulation and integrin $\beta 4$ neoexpression} suppress caspase- 3 activity in fibroblasts

Anoikis is a form of caspase-dependent apoptosis that is caused by loss of integrin binding. Because caspase- 3 is a key effector in the extrinsic apoptotic pathway, we examined caspase- 3 activity in fibroblasts in the absence or presence of cancer cell CM for 72 hours. Without integrin $\beta 4$ expression, InFs had lower caspase- 3 activity than did CAFs and NBFs, and MDAMB-231 CM further reduced caspase-3 activity, whereas MCF7 CM had no effect. In addition, the induction of integrin $\beta 4$ dramatically reduced caspase3 activity, which also became much less dependent on MDA-MB-231 CM stimulation. The lowest measured caspase- 3 activity was in InFs/ $\beta 4$ cells stimulated with MDA-MB-231 CM (Figure 4A). To confirm whether the caspase- 3 activity of fibroblasts is regulated by the binding of laminin-332 to integrin $\alpha 3 \beta 1$ or $\alpha 6 \beta 4$, InFs and InFs $/ \beta 4$ cells were treated with blocking antibodies against integrin $\beta 1, \beta 4$, or laminin-332, and their caspase- 3 activity was compared. Inhibition of integrin $\beta 1$ significantly inhibited caspase- 3 activity in InFs and InFs $/ \beta 4$, whereas inhibition of integrin $\beta 4$ and laminin332 had no effect.

Similar to these results, induction of integrin $\beta 4$ also reduced caspase- 3 activity (Figure 4B). To show further that the caspase- 3 activity of fibroblasts was decreased by laminin-332 upregulation, CAFs (Ln-332-low) and CAFs $/ \beta 4$ were treated with $10 \mu M$ purified laminin332 , and the resulting caspase- 3 activities were measured. Treatment with laminin-332 significantly decreased caspase- 3 activity in both CAFs and CAFs/ $\beta 4$ (Figure 4C).

\section{Enhancement of anoikis resistance by integrin $\beta 4$ neoexpression is mediated by Rac1 activation}

Akt phosphorylation and Rac1 activation are associated with integrin-mediated anoikis resistance. In the absence of stimulation from cancer cells, InFs showed a higher level of phosphorylation of Akt (Ser473) than did CAFs and NBFs. Akt phosphorylation was enhanced by MDAMB-231 CM and decreased by MCF7 CM (Figure 5A). GTP-bound Rac1 was downregulated in InFs but upregulated in CAFs and NBFs, and MCF7 CM further increased GTP-bound Rac1 in InFs (Figure 5A). Induction of integrin $\beta 4$ expression dramatically increased GTP-bound Rac1, independent of stimulation from cancer cells, but did not have a significant effect on Akt phosphorylation (Figure 5B).

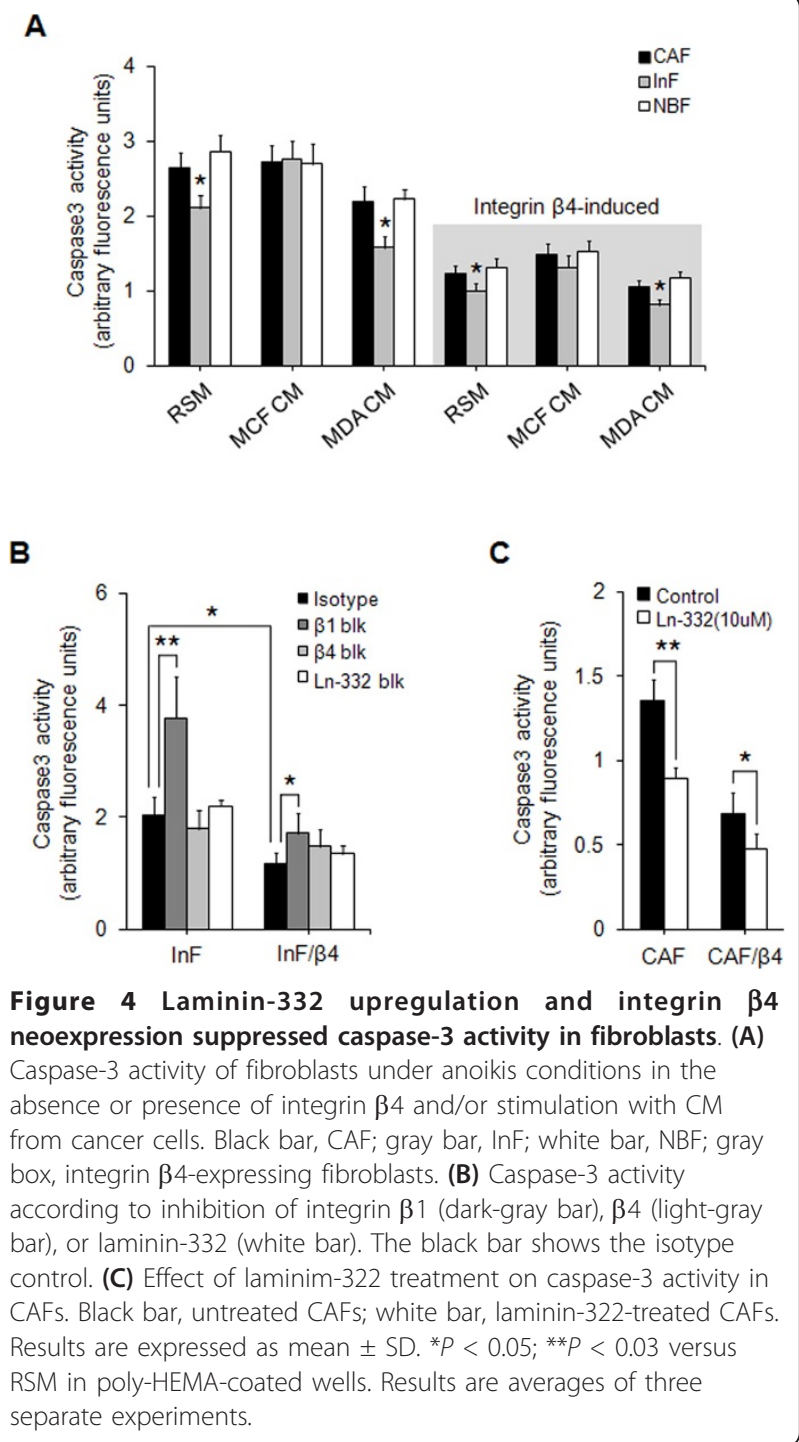

Integrin $\beta 4$ neoexpression suppresses aggregation of fibroblasts under anoikis conditions

Synoikis, the formation of cell aggregates in nonadherent conditions, has been proposed as a mechanism by which cells overcome anoikis. Therefore, we investigated the role of synoikis in the anoikis resistance of fibroblasts and whether it is affected by laminin-332 upregulation or integrin $\beta 4$ neoexpression. In the absence of integrin $\beta 4$ expression, the fibroblasts aggregated and formed big clusters under anoikis conditions; this aggregation was not affected by the upregulation of laminin332 by MDA-MB-231 CM (Figure 6A). In contrast, integrin $\beta 4$ expression suppressed fibroblast aggregation independent of laminin-332 (Figure 6B). To confirm whether fibroblast aggregation is caused by integrin $\beta 4$ expression, rather than binding of laminin-332 to integrins, we treated fibroblasts with blocking antibodies 


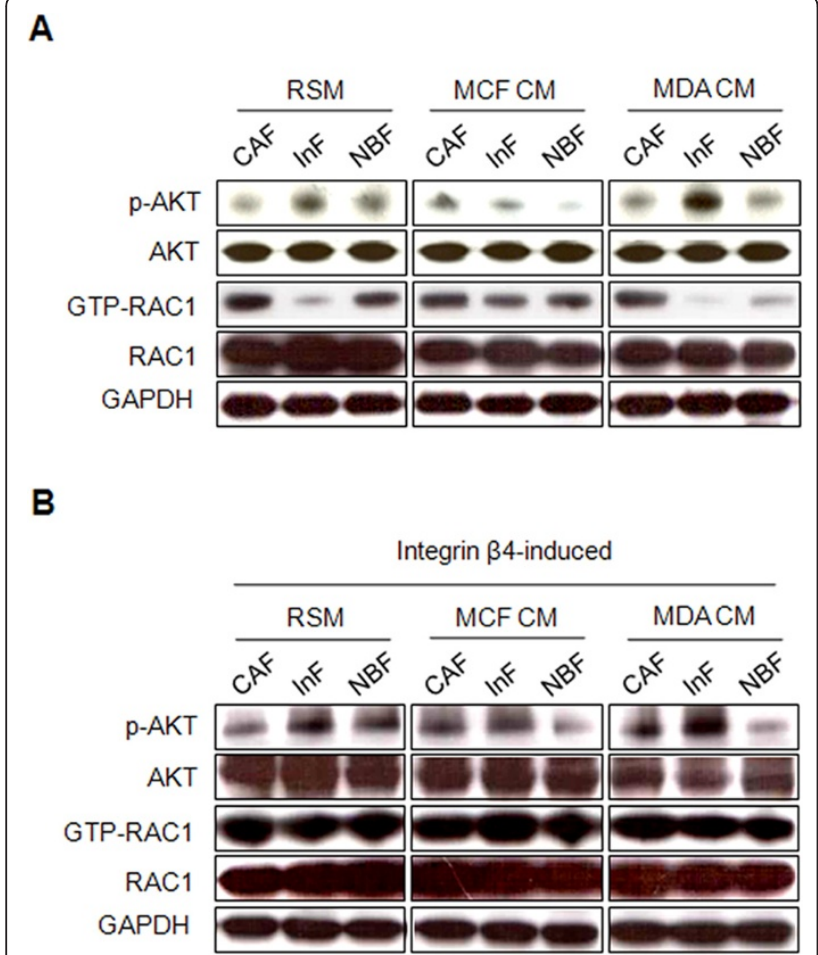

Figure 5 Integrin $\beta 4$ neoexpression induces Rac1 activation. Akt phosphorylation and Racl activation is shown in (A) wild-type and (B) integrin $\beta 4$-expressing fibroblasts. Wild-type and integrin $\beta 4$-expressing fibroblasts were incubated in RSM, MCF7 CM, or MDA-MB-231 CM under anoikis conditions for 24 hours. Suspended fibroblasts were harvested for Rac1 pull-down assays.

against integrin $\beta 1$ or $\beta 4$. Neither blocking antibody affected fibroblast aggregation (Figure $6 \mathrm{C}$ ). Based on these findings, we suggest that integrin $\beta 4$ suppresses fibroblast aggregation in anoikis conditions. Synoikis is known to depend on E-cadherin-mediated cell-cell adhesion; therefore, the suppressive effect of integrin $\beta 4$ expression on fibroblast aggregation may be caused by a decrease in E-cadherin expression. However, the fibroblasts expressing integrin $\beta 4$ did not show decreased Ecadherin expression compared with wild-type controls. Because $\beta$-catenin displays E-cadherin on the cell surface, we postulated that downregulation of $\beta$-catenin may be the cause of defective E-cadherin-mediated cellcell interaction. Consistent with this notion, $\beta$-catenin expression was decreased in fibroblasts expressing integrin $\beta 4$ (Figure 6D).

\section{Discussion}

In the present work, we hypothesized that myofibroblasts gain an anoikis-resistant phenotype during tissue remodeling through laminin-332 upregulation and integrin $\beta 4$ neoexpression induced by interaction with invasive breast cancer cells. To prove our hypothesis, we isolated three types of fibroblasts, CAFs from the tumor zone (the epicenter of tumor burden), InFs from the interface zone (fibrosis), and NBFs from the normal zone (distal normal tissue) of IDC, and co-cultured them with tumor cells to investigate the tumor-stroma interaction. InFs, the myofibroblasts undergoing tissue remodeling, showed a higher resistance to anoikis than did CAFs and NBFs as a result of their high endogenous expression level of laminin-332 and its dramatic upregulation by conditioned medium from invasive MDA-MB231 cancer cells. In addition, integrin $\beta 4$ neoexpression was induced in fibroblasts by cell-to-cell contact with MDA-MB-231 cells regardless of the type of fibroblast, and expression of integrin $\beta 4$ increased anoikis resistance to a level approximately twofold higher than that induced by laminin-332 upregulation. From these results, we conclude that invasive breast cancer cells confer an anoikis-resistant phenotype on myofibroblasts during tissue remodeling by inducing laminin-332 upregulation and integrin $\beta 4$ neoexpression, and that InFs may be the primary myofibroblasts involved in tissue remodeling during invasion of breast cancer cells.

This may be the first study to describe how myofibroblasts acquire an anoikis-resistant phenotype during tissue remodeling by using a model of invasive breast cancer. Previously, one group reported that transforming growth factor $\beta 1$ (TGF- $\beta 1$ ) confers an anoikis-resistant phenotype on normal primary human fetal lung fibroblasts [25], and another group showed that the matricellular protein TSP1 induces anoikis resistance in mouse embryonic fibroblasts [21]. However, the fibroblasts used in these studies were not derived from tumor stroma, and we believed that it was necessary to investigate the development of anoikis-resistant myofibroblasts in the context of tumor progression. Therefore, in this study, we used IDC to examine the development of anoikis-resistant myofibroblasts during tissue remodeling.

We chose to study IDC for two reasons: the first was the dense fibrosis around the tumor burden, which is a phenotype of vigorous tissue remodeling [7-9], and the second was aberrant upregulation of laminin-332 in the fibrosis (in contrast, laminin-332 upregulation was not detected in its counterpart, DCIS) [13]. Because laminin-332 is widely known to promote cell survival, the anoikis resistance of myofibroblasts is likely mediated by laminin-332-dependent signaling. To test this assumption, we first investigated laminin-322 expression in CAFs, InFs, and NBFs and found that the expression pattern in the fibroblasts was the same as that in situ (Figure 1A). Based on $\alpha$-SMA expression and their location in the tissue, CAFs and InFs are presumed to be the myofibroblasts that interact with invasive tumor cells during tissue remodeling. 


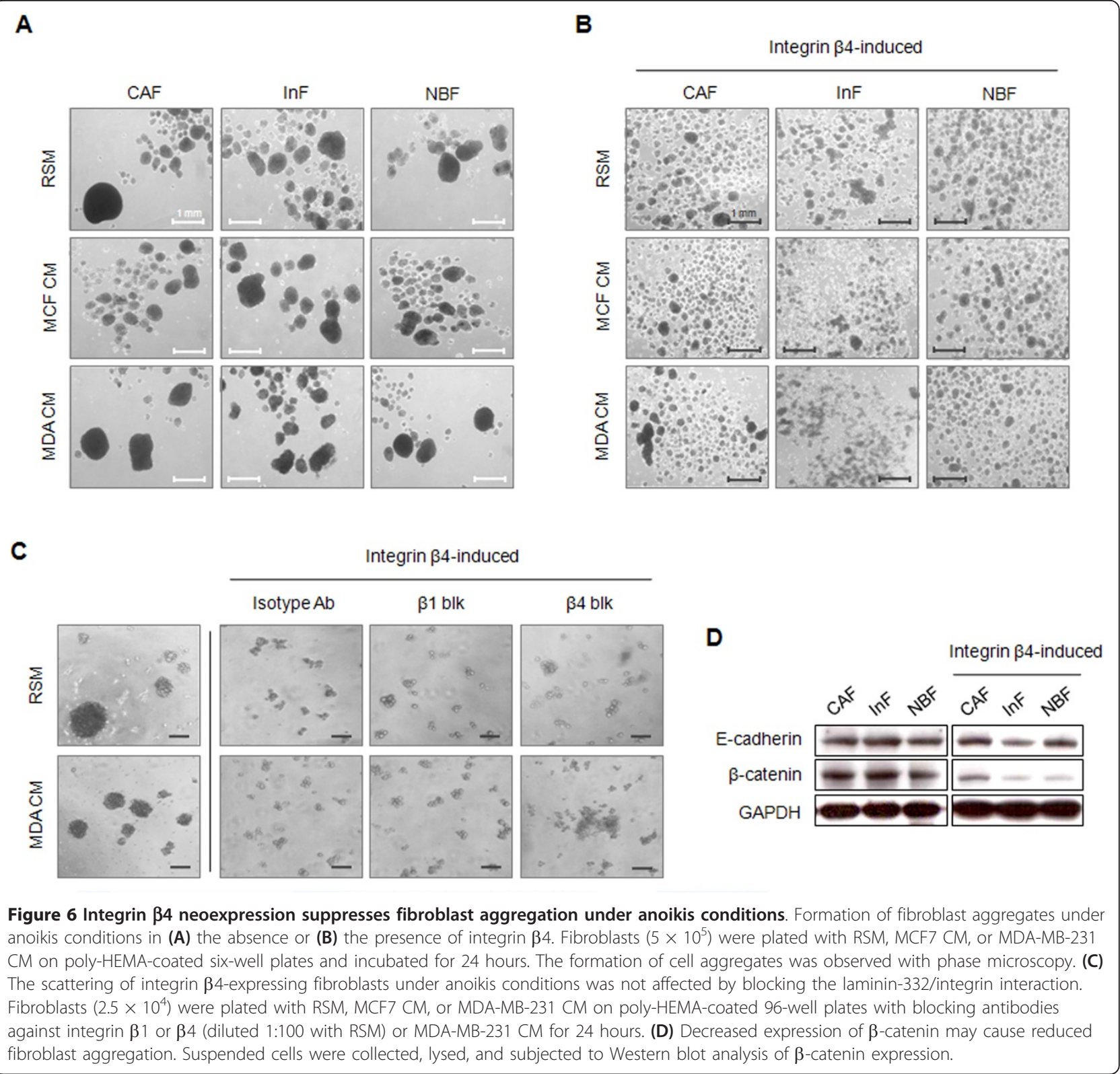

Within the interface zone (IZ) adjacent to the tumor zone (TZ), invasive breast cancer cells may interact with InFs via cell-to-cell contact at the border between the $\mathrm{TZ}$ and IZ or with diffusible factors away from the border and within the IZ. It is therefore possible that the expression of laminin-332 or its receptors, integrin $\alpha 3 \beta 1$ and $\alpha 6 \beta 4$, is affected differently according to the type of interaction. To test this notion, fibroblasts were co-cultured with tumor cells to simulate cell-to-cell contact or were stimulated with the CM from tumor cells to test the role of diffusible factors. In our previous study, laminin-332 upregulation in the IZ was shown to be associated with tumor invasiveness [13]. Thus, MDA-MB231 and MCF7 were used as invasive and noninvasive breast cancer cell types, respectively. After treatment with MDA-MB-231 CM, but not MCF7 CM, laminin$\gamma 2$, a subunit used to identify laminin-332, was upregulated in InFs, and this upregulation was maintained under anoikis conditions. Expression of laminin- $\gamma 2$ in CAFs and NBFs was also promoted by MDA-MB-231 $\mathrm{CM}$, but to a lesser extent than in InFs (Figure 1C). The degree of laminin- $\gamma 2$ induction by diffusible factors produced by MDA-MB-231 seemed to depend on the endogenous capacity of fibroblasts to express laminin- $\gamma 2$. According to previous reports, TGF- $\beta$ is a major soluble factor produced by MDA-MB-231 [26-28]. Therefore, we examined whether laminin $-\gamma 2$ upregulation by MDAMB-231 CM is induced by TGF- $\beta$ and found that the 
TGF- $\beta$ inhibitor LY2157299 effectively suppressed laminin- $\gamma 2$ expression (see Additional file 1, Figure S1). Based on this result, we suggest that expression of laminin-322 is at least partially induced by TGF- $\beta$ secreted by invasive breast cancer cells. However, further evaluation is required because we did not test other cytokines that are highly secreted from invasive breast cancer cells. In contrast to laminin-332 upregulation by MDAMB-231 CM, integrins $\alpha 3, \alpha 6$, and $\beta 1$ were highly expressed in fibroblasts and not affected by either diffusible factors or direct contact with MDA-MB-231 cells (Figure 2, and see Additional file 1, Figure S3). Integrin $\beta 4$ was not endogenously expressed in fibroblasts, but its expression was newly induced by direct contact with MDA-MB-231 cells. Almost no difference was found in the induction of anoikis resistance by integrin $\beta 4$ neoexpression in the different types of fibroblasts.

When in direct contact with invasive breast cancer cells, the anoikis resistance of InFs involved both integrin $\alpha 3 \beta 1$ and integrin $\alpha 6 \beta 4$ as a result of integrin $\beta 4$ neoexpression. Although both integrins are known receptors for laminin-332, their signaling pathways, signal duration, and dependency on laminin-332 are different. In the case of integrin $\alpha 3 \beta 1$, signaling was dependent on laminin-332 binding and mediated by Akt (Ser473) phosphorylation (Figure 5A). In contrast, integrin $\alpha 6 \beta 4$ mediated signaling was independent of laminin-332 and mediated through Rac1 activation (Figure 5B). Anoikis resistance induced by integrin $\alpha 6 \beta 4$ was approximately twofold higher than that induced by integrin $\alpha 3 \beta 1$ (Figure 3D). This is supported by a report that Rac1 activation through integrin $\alpha 6 \beta 4$ produces a long-lasting survival signal [18]. Furthermore, the long cytoplasmic tail of integrin $\beta 4$ can be phosphorylated by other factors such as c-Met, independent of laminin-332 [29]. In addition to anoikis resistance, integrin $\beta 4$ neoexpression suppressed fibroblast aggregation under anoikis conditions, possibly as a result of downregulation of $\beta$-catenin (Figure 6D). Many cells overcome anoikis through synoikis, the formation of cell clusters via E-cadherin-mediated

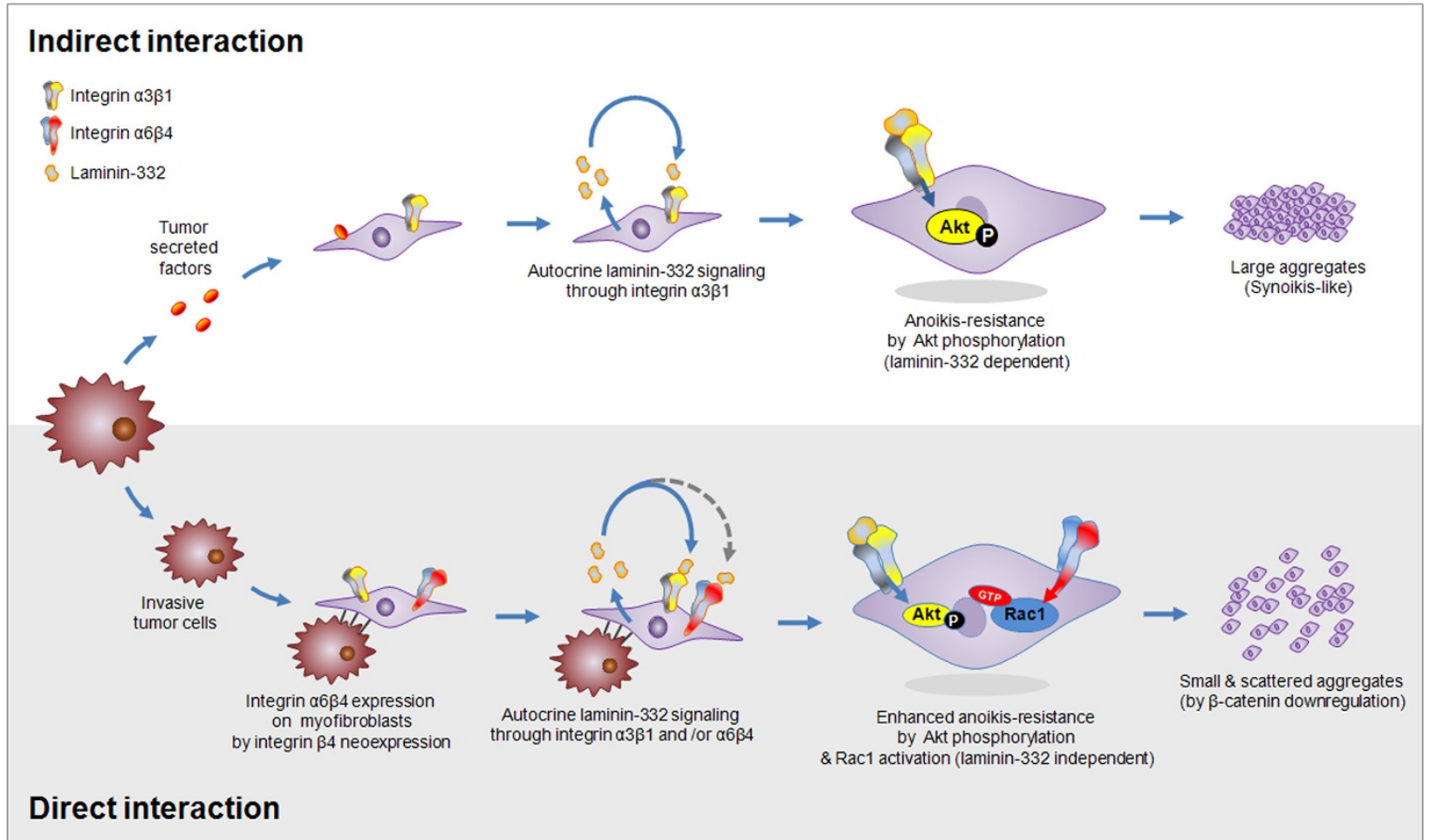

Figure 7 Two possible mechanisms by which myofibroblasts acquire anoikis resistance via integrin receptors for laminin-332 during tissue remodeling. Direct and indirect interactions may occur between invasive tumor cells and myofibroblasts in the interface zone of invasive ductal carcinoma. Myofibroblasts within the interface zone could be stimulated to express laminin-332 by diffusible factors from invasive breast cancer cells. Binding of laminin-332 to integrin $\alpha 3 \beta 1$ on the myofibroblasts turns on a cell-survival signaling pathway mediated by Akt phosphorylation. In this case, myofibroblasts may simultaneously use synoikis to overcome anoikis. In addition, in the border between the tumor zone and the interface zone, invasive breast cancer cells can directly interact with myofibroblasts, leading to integrin $\beta 4$ neoexpression. Once integrin $\beta 4$ is induced, myofibroblasts use both integrin $\alpha 3 \beta 1$ and $\alpha 6 \beta 4$ as receptors for laminin-332, and become resistant to anoikis via Akt phosphorylation (laminin-332 dependent) and Rac1 activation (laminin-332 independent). Moreover, integrin $\beta 4$ neoexpression may inhibit myofibroblast aggregation through downregulation of $\beta$-catenin. 
interaction between cells. The expression of E-cadherin on the cell surface is known to be regulated by $\beta$-catenin [30]; therefore, decreased expression of $\beta$-catenin by integrin $\beta 4$ neoexpression may cause defective surface expression of E-cadherin, which in turn suppresses fibroblast aggregation. Considering a recent report that myofibroblasts in the primary tumor site metastasize to the lung along with tumor cells [31], in the context of tumor metastasis, single cells or a small aggregate of myofibroblasts may be more likely to undergo distant migration with invasive tumor cells than might a large cluster.

In summary, two mechanisms seem to exist by which myofibroblasts acquire anoikis resistance through laminin-integrin signaling during tissue remodeling as a result of either direct or indirect interaction between invasive breast cancer cells and myofibroblasts in the fibrosis (or interface zone) (Figure 7). For indirect interaction, factor(s) secreted from invasive tumor cells stimulate myofibroblasts to overexpress laminin-332. Laminin-332 binds to integrin $\alpha 3 \beta 1$ on myofibroblasts and turns on an autocrine cell survival signal mediated by Akt (Ser473) phosphorylation. In addition, myofibroblasts that lose their attachment spontaneously use synoikis to overcome anoikis.

Second, direct contact between invasive breast cancer cells and myofibroblasts induces integrin $\beta 4$ neoexpression in the fibroblasts. Consequently, myofibroblasts express both integrin $\alpha 3 \beta 1$ and integrin $\alpha 6 \beta 4$ as receptors for laminin-332. In this case, cell-survival signals are mediated by Rac1 activation and Akt (Ser473) phosphorylation. Akt (Ser473) phosphorylation requires binding of laminin-332 to integrin $\alpha 3 \beta 1$, whereas Rac1 activation by integrin $\alpha 6 \beta 4$ is not dependent on laminin-332. Furthermore, integrin $\beta 4$ neoexpression suppresses fibroblast aggregation under anoikis conditions, possibly through downregulation of $\beta$-catenin.

\section{Conclusions}

Invasive breast cancer cells confer an anoikis-resistant phenotype on myofibroblasts during tissue remodeling by inducing laminin-332 upregulation and integrin $\beta 4$ neoexpression. Our findings indicate that interface fibroblasts may be the primary myofibroblasts involved in tissue remodeling during breast cancer invasion.

\section{Additional material}

Additional file 1: Supplementary Figures 1, 2, 3, and 4. Figure S1. TGF- $\beta$ in MDA-MB-231 CM causes laminin-332 upregulation in InFs. Figure S2. Endogenous expression of integrins $\alpha 3$ and $\alpha 6$ in IDC fibroblasts. Figure S3. Expression of integrins $\beta 1$ and $\beta 4$ is not affected by diffusible factors from breast cancer cells. Figure S4. Exogenous expression of integrin $\beta 4$ in IDC fibroblasts by transfection.

\section{Abbreviations}

CAF: cancer-associated fibroblast (fibroblasts isolated from the TZ); CMFDA: (5-chloromethylfluorescein diacetate); DCIS: ductal carcinoma in situ; ECM: extracellular matrix; IDC: invasive ductal carcinoma; InF: interface fibroblast (fibroblasts isolated from the IZ); IZ: interface zone (tumor-free fibrotic zone adjacent to tumor burden); Ln-332: laminin-332; NBF: normal breast fibroblast (fibroblasts isolated from the NZ); NZ: normal zone (normal tissue neighboring the IZ); Poly-HEMA: poly(2-hydroxyethyl methacrylate); TZ: tumor zone (tumor burden of IDC).

\section{Acknowledgements}

This study was supported by the Korea Healthcare Technology R\&D Project, Ministry for Health, Welfare and Family Affairs, Republic of Korea (CNH A101264) and by a faculty research grant of Yonsei University College of Medicine for 2011(6-2011-0106; CNH).

\section{Author details}

${ }^{1}$ Brain Korea 21 Project for Medical Science, Yonsei University College of Medicine, 134 Sinchon-dong, Seodaemun-gu, Seoul, 120-752, South Korea. ${ }^{2}$ Severance Biomedical Science Institute (SBSI), Yonsei University College of Medicine, 134 Sinchon-dong, Seodaemun-gu, Seoul, 120-752, South Korea. ${ }^{3}$ Department of Pathology, Yonsei University College of Medicine, 134 Sinchon-dong, Seodaemun-gu, Seoul, 120-752, South Korea. ${ }^{4}$ Global 5-5-10 System Biology, Yonsei University, 50 Yonsei-ro, Seodaemun-gu, Seoul, 120749, South Korea.

\section{Authors' contributions}

BGK developed the concept and design of the study, performed the experiments, analyzed and organized the data, and wrote the manuscript. MQG carried out the isolation of fibroblasts, maintained them for subsequent experiments, and helped draft the manuscript. YPC performed cell sorting after co-culture, and participated in the design of the study. SK was involved in the anoikis assay. HRP and KSK participated in immunoassay. $\mathrm{NHC}$ participated in the selection of patient breast tissue material, the conception, design and coordination of the study, and helped draft the manuscript. All authors read and approved the final manuscript.

\section{Competing interests}

We declare no conflicts of interest in all participants.

Received: 1 February 2012 Revised: 10 May 2012

Accepted: 6 June 2012 Published: 6 June 2012

\section{References}

1. Johnsen M, Lund LR, Romer J, Almholt K, Dano K: Cancer invasion and tissue remodeling: common themes in proteolytic matrix degradation. Curr Opin Cell Biol 1998, 10:667-671.

2. Fattman CL: Apoptosis in pulmonary fibrosis: too much or not enough? Antioxidants Redox Signal 2008, 10:379-385.

3. Maher TM, Evans IC, Bottoms SE, Mercer PF, Thorley AJ, Nicholson AG, Laurent GJ, Tetley TD, Chambers RC, McAnulty RJ: Diminished prostaglandin E2 contributes to the apoptosis paradox in idiopathic pulmonary fibrosis. Am J Respir Crit Care Med 2010, 182:73-82.

4. Jinta T, Miyazaki Y, Kishi M, Akashi T, Takemura T, Inase N, Yoshizawa Y: The pathogenesis of chronic hypersensitivity pneumonitis in common with idiopathic pulmonary fibrosis: expression of apoptotic markers. Am J Clin Pathol 2010, 134:613-620.

5. Uhal BD: Apoptosis in lung fibrosis and repair. Chest 2002, 122:(6 Suppl):293S-298S.

6. Radisky DC, Przybylo JA: Matrix metalloproteinase-induced fibrosis and malignancy in breast and lung. Proc Am Thorac Soc 2008, 5:316-322.

7. Kawashiri S, Tanaka A, Noguchi N, Hase T, Nakaya H, Ohara T, Kato K, Yamamoto E: Significance of stromal desmoplasia and myofibroblast appearance at the invasive front in squamous cell carcinoma of the oral cavity. Head Neck 2009, 31:1346-1353.

8. Ronnov-Jessen L, Petersen OW, Bissell MJ: Cellular changes involved in conversion of normal to malignant breast: importance of the stromal reaction. Physiol Rev 1996, 76:69-125.

9. Dvorak HF: Tumors: wounds that do not heal: similarities between tumor stroma generation and wound healing. N Engl J Med 1986, 315:1650-1659. 
10. Tomasek JJ, Gabbiani G, Hinz B, Chaponnier C, Brown RA: Myofibroblasts and mechano-regulation of connective tissue remodelling. Nat Rev Mol Cell Biol 2002, 3:349-363.

11. Hinz B, Phan SH, Thannickal VJ, Galli A, Bochaton-Piallat ML, Gabbiani G: The myofibroblast: one function, multiple origins. Am J Pathol 2007, 170:1807-1816

12. Giancotti FG: Integrin signaling: specificity and control of cell survival and cell cycle progression. Curr Opin Cell Biol 1997, 9:691-700.

13. Kim BG, An HJ, Kang S, Choi YP, Gao MQ, Park H, Cho NH: Laminin-332rich tumor microenvironment for tumor invasion in the interface zone of breast cancer. Am J Pathol 2011, 178:373-381.

14. Martin KJ, Kwan CP, Nagasaki K, Zhang X, O'Hare MJ, Kaelin CM, Burgeson RE, Pardee $A B$, Sager R: Down-regulation of laminin-5 in breast carcinoma cells. Mol Med 1998, 4:602-613.

15. Manohar A, Shome SG, Lamar J, Stirling L, lyer V, Pumiglia K, DiPersio CM: Alpha 3 beta 1 integrin promotes keratinocyte cell survival through activation of a MEK/ERK signaling pathway. J Cell Sci 2004, 117:4043-4054.

16. Sehgal BU, DeBiase PJ, Matzno S, Chew TL, Claiborne JN, Hopkinson SB, Russell A, Marinkovich MP, Jones JC: Integrin beta4 regulates migratory behavior of keratinocytes by determining laminin-332 organization. Biol Chem 2006, 281:35487-35498

17. Choma DP, Milano V, Pumiglia KM, DiPersio CM: Integrin alpha3beta1dependent activation of FAK/Src regulates Rac1-mediated keratinocyte polarization on laminin-5. J Invest Dermatol 2007, 127:31-40.

18. Zahir N, Lakins JN, Russell A, Ming W, Chatterjee C, Rozenberg Gl, Marinkovich MP, Weaver VM: Autocrine laminin-5 ligates alpha6beta4 integrin and activates RAC and NFkappaB to mediate anchorageindependent survival of mammary tumors. J Cell Biol 2003, 163:1397-1407.

19. Fujisaki $H$, Hattori S: Keratinocyte apoptosis on type I collagen gel caused by lack of laminin 5/10/11 deposition and Akt signaling. Exp Cell Res 2002, 280:255-269.

20. Nguyen BP, Ryan MC, Gil SG, Carter WG: Deposition of laminin 5 in epidermal wounds regulates integrin signaling and adhesion. Curr Opin Cell Biol 2000, 12:554-562.

21. Pallero MA, Elzie CA, Chen J, Mosher DF, Murphy-Ullrich JE: Thrombospondin 1 binding to calreticulin-LRP1 signals resistance to anoikis. FASEB J 2008, 22:3968-3979.

22. Gao MQ, Kim BG, Kang S, Choi YP, Park H, Kang KS, Cho NH: Stromal fibroblasts from the interface zone of human breast carcinomas induce an epithelial-mesenchymal transition-like state in breast cancer cells in vitro. J Cell Sci 2010, 123:3507-3514.

23. Olumi AF, Grossfeld GD, Hayward SW, Carroll PR, TIsty TD, Cunha GR: Carcinoma-associated fibroblasts direct tumor progression of initiated human prostatic epithelium. Cancer Res 1999, 59:5002-5011.

24. Dans M, Gagnoux-Palacios L, Blaikie P, Klein S, Mariotti A, Giancotti FG: Tyrosine phosphorylation of the beta 4 integrin cytoplasmic domain mediates Shc signaling to extracellular signal-regulated kinase and antagonizes formation of hemidesmosomes. J Biol Chem 2001, 276:1494-1502.

25. Horowitz JC, Rogers DS, Sharma V, Vittal R, White ES, Cui Z, Thannickal VJ: Combinatorial activation of FAK and AKT by transforming growth factorbeta1 confers an anoikis-resistant phenotype to myofibroblasts. Cell Signal 2007, 19:761-771.

26. Arrick BA, Korc M, Derynck R: Differential regulation of expression of three transforming growth factor beta species in human breast cancer cell lines by estradiol. Cancer Res 1990, 50:299-303.

27. Zajchowski D, Band V, Pauzie N, Tager A, Stampfer M, Sager R: Expression of growth factors and oncogenes in normal and tumor-derived human mammary epithelial cells. Cancer Res 1988, 48:7041-7047.

28. Dickson RB, Bates SE, McManaway ME, Lippman ME: Characterization of estrogen responsive transforming activity in human breast cancer cell lines. Cancer Res 1986, 46:1707-1713.

29. Chan PC, Chen SY, Chen CH, Chen HC: Crosstalk between hepatocyte growth factor and integrin signaling pathways. J Biomed Sci 2006 13:215-223.

30. Fukunaga Y, Liu H, Shimizu M, Komiya S, Kawasuji M, Nagafuchi A: Defining the roles of beta-catenin and plakoglobin in cell-cell adhesion: isolation of beta-catenin/plakoglobin-deficient F9 cells. Cell Struct Funct 2005, 30:25-34.
31. Duda DG, Duyverman AM, Kohno M, Snuderl M, Steller EJ, Fukumura D, Jain RK: Malignant cells facilitate lung metastasis by bringing their own soil. Proc Natl Acad Sci U S A 2010, 107:21677-21682.

doi: $10.1186 /$ bcr3203

Cite this article as: Kim et al:: Invasive breast cancer induces laminin332 upregulation and integrin $\beta 4$ neoexpression in myofibroblasts to confer an anoikis-resistant phenotype during tissue remodeling. Breast Cancer Research 2012 14:R88.

\section{Submit your next manuscript to BioMed Central and take full advantage of:}

- Convenient online submission

- Thorough peer review

- No space constraints or color figure charges

- Immediate publication on acceptance

- Inclusion in PubMed, CAS, Scopus and Google Scholar

- Research which is freely available for redistribution

Submit your manuscript at www.biomedcentral.com/submit
Ciomed Central 